\title{
Cambios en la productividad y sus determinantes en explotaciones lecheras de Cuba
}

\author{
J. Flores ${ }^{*}, 1$, J. Herrera-Toscano ${ }^{* *}$ y S. Flores ${ }^{* * *}$
}

* UNELLEZ-Guanare. Antiguo Convento de San Francisco, carrera 3 entre carrera 16 y 17, Mesa de Cavaca, Guanare, Estado Portuguesa. Venezuela

** Departamento de Manejo y Alimentación de Rumiantes, Instituto de Ciencia Animal. Carretera Central, Km 47 1/2. San José de las Lajas, Mayabeque. Código Postal: 32700. República de Cuba

*** Depto. Ingeniería de Organización, Administración de Empresa y Estadística, E.T.S. Ingenieros Industriales, Universidad Politécnica de Madrid. C/ José Gutiérrez Abascal, 2. 28006 Madrid, España

\section{Resumen}

El objetivo de este artículo es estudiar los cambios en la productividad y sus elementos determinantes -cambio técnico y cambio en eficiencia- de una muestra de explotaciones lecheras cubanas ubicadas en el municipio de San José de las Lajas. Con este propósito se calculan índices Malmquist de productividad con técnicas DEA y datos correspondientes al período 2006-08. Asimismo, se asumen rendimientos variables a escala y se utiliza una orientación al producto. Se consideraron dos insumos y dos productos. Los resultados indican que debido a una caída de la productividad de las explotaciones lecheras durante el periodo 2006-2007, que no fue compensada por el incremento ocurrido durante el lapso 20072008, todo el periodo de estudio, del 2006 al 2008, reflejó una pequeña reducción de la productividad, que fue ocasionada en su totalidad por un ligero retroceso tecnológico. Este resultado es explicado, parcialmente, por un aumento del número de vacas en el año 2007 que no se expresó proporcionalmente en la producción correspondiente a ese año.

Palabras clave: Malmquist, eficiencia, cambio tecnológico.

\section{Abstract \\ Changes in productivity and its determinants in Cuba dairy farms}

The objective of this paper is to study changes in productivity and its determinants-technical change and efficiency change-of a sample of Cuban dairy farms located in the municipality of San Jose de las Lajas. For this purpose Malmquist productivity indexes calculated with DEA techniques and data for the period 2006-08. Also, assume variable returns to scale and used a product orientation. We considered two inputs and two outputs. The results indicate that due to a drop in productivity of dairy farms in 20062007, which was not offset by the increase occurred during the period 2007-2008, the entire study period from 2006 to 2008, showed a small reduction productivity, which was caused entirely by a slight drop technology. This result is explained, in part, by an increase in the number of cows in 2007 that proportion was not expressed in the production for that year.

Key words: Malmquist, efficiency, change technological.

1. Autor para correspondencia: joseovidioflores@gmail.com, http://joseovidioflores.wordpress.com/ http://dx.doi.org/10.12706/itea.2014.012 


\section{Introducción}

El desarrollo del sector lechero cubano no se desmarca de su particular sistema político ni de la existencia de cincuenta años de bloqueo por parte de Estados Unidos (Ponce, 2009). Ello se puede resumir en que, la leche es un producto de alta sensibilidad para la población, ya que se destina a mujeres gestantes, ancianos y enfermos, con un precio altamente subsidiado. Constituye uno de los pocos productos agrícolas que tiene precios regulados para el productor y se comercializa únicamente con la participación del estado. Cuba tiene 11.3 millones de habitantes y una población flotante de dos millones de personas anuales, fundamentalmente turistas. Existe una población de cuatro millones de cabezas de bovinos, 45 mil búfalos y $200 \mathrm{mil}$ cabras. Cerca de 200 mil familias están vinculadas a la actividad lechera. La producción registrada en el año 2006 fue de 371,7 millones de litros de leche (ML), al año siguiente fue de 411,3 ML y en el 2008 se registraron 399,9 ML (aunque se estimaron $480 \mathrm{ML}$ ) mientras que, para estos mismos años, las vacas en ordeño (en miles de cabezas) fueron 317,$6 ; 318,2$ y 275,1 , respectivamente (Nova 2008; Ponce, 2009).

Aunque los promedios nacionales de los indicadores productivos y reproductivos de la ganadería vacuna en Cuba son deficientes, el sector campesino ha mostrado índices muy favorables, sobre todo en las últimas dos décadas (Miranda et al., 2012). Por citar un ejemplo, en el año 2008 , con solo el $27 \%$ de la superficie agrícola nacional y el $55 \%$ del rebaño vacuno, el sector campesino entregó el $62 \%$ de la leche y el $45 \%$ de la carne vacuna producida nacionalmente (Mirabal, 2010). En el año siguiente, después del comienzo del proceso de entrega de tierras en usufructo, este sector campesino produjo el $96 \%$ del tabaco, el $70 \%$ de la carne de cerdo, el $60 \%$ de las viandas y hortalizas, y el $59 \%$ de la leche de vaca, además posee el $90 \%$ del ganado menor y el $62 \%$ de la masa vacuna (González, 2009).

Para el mejoramiento de la ganadería cubana, Senra et al. (2010) recomiendan la utilización de metodologías -a mediano y largo plazopara evaluar los resultados finales de la aplicación de las tecnologías. Es conveniente garantizar el control y ajuste sistemáticos, durante las etapas iniciales de su aplicación para evitar las pérdidas que conduzcan al deterioro paulatino de la ganadería, como en décadas anteriores.

Precisamente, una forma de medir el impacto en el uso de las tecnologías consiste en estimar la productividad en el tiempo. Para ello, existen varios métodos de medición como la contabilidad del crecimiento y los números índices. Sin embargo, estos enfoques adolecen de varias limitaciones, entre las que destacan la asunción de que todas las unidades de producción son plenamente eficientes y los requerimientos de información de los precios que pueden no estar siempre disponibles. Por el contrario, el índice de productividad de Malmquist (IPM) desarrollado por Färe et al. (1994), al estar basado en el método DEA (Data Envelopment Analysis) presenta importantes ventajas que superan sus desventajas (Schuschny, 2007). El IPM es un índice basado en ratios de funciones de distancia, que sirve para medir el cambio productivo que experimenta cualquier empresa que transforme recursos. Dicho cambio se puede descomponer en cambios de naturaleza técnica y cambios en la eficiencia o catching-up.

En el caso del sector pecuario, la productividad puede ser incrementada de dos maneras: mediante el progreso tecnológico y a través de la mejora de la eficiencia técnica. La medición y el monitoreo de estas dos medidas del crecimiento de la productividad puede ayudar, de manera muy efectiva, para formular y ejecutar políticas públicas agrarias ten- 
dientes a mejorar el sector. El cambio tecnológico (o progreso tecnológico) se produce por el desarrollo y adopción de nuevas tecnologías de producción o de administración, y es una de las vías para que las mejores fincas se superen a sí mismas.

Por otra parte, los cambios en la eficiencia técnica representan la tasa de adopción de las tecnologías disponibles, o la tasa a la cual las fincas promedio alcanzan los desempeños (o logros) de las mejores fincas (Gray et al., 2011). Por lo general, estos cambios están asociados al factor humano y representan una mejora en las prácticas agrarias relacionadas con los procesos productivos o administrativos de las fincas ineficientes en comparación con sus pares eficientes, ubicados en la frontera de producción.

Los cambios y tendencias en la productividad agrícola han sido de gran interés para aquellas personas relacionadas con la agricultura, debido, principalmente, a las razones siguientes: a) el crecimiento de la productividad es el motor del cambio estructural en la producción, b) la necesidad de incrementar los niveles de alimentos, fibras y combustibles debido a un aumento de la población, c) una base fija o declinante del recurso tierra, d) cambios en los insumos y métodos de producción, y e) cambios reales y potenciales en las políticas nacionales y en las reglas internacionales de comercio, entre otras.

Como es lógico suponer, dada la gran cantidad de variables que inciden en la productividad agraria, los resultados de las aplicaciones empíricas son dispares, ya que en algunas se reportaron crecimientos de la productividad y en otras se obtuvieron evidencias de un relativo estancamiento, e incluso disminuciones de la productividad. En el primer grupo el crecimiento se debió, principalmente, a una de las dos fuentes de productividad: a mejoras en la eficiencia técnica o al progreso tecnológico, según se desprende de los trabajos de Tauer (1998), Graham (2009), Luik et al. (2011) y Zuniga (2010), entre otros. En el segundo grupo se ubican los trabajos de Karanja et al. (2012) y Singh y Singh (2012).

Estas discrepancias en los resultados empíricos es una importante motivación para continuar esta línea de investigación, a fin de contribuir a explicar la realidad de los procesos productivos agrarios. Por ello, el presente estudio, con base a una muestra de 24 explotaciones lecheras del municipio San José de las Lajas, Cuba, tiene como objetivo analizar la productividad agraria, separándola en los componentes de cambio de eficiencia técnica y cambio tecnológico, medida durante el periodo 2006 a 2008. Se empleó el índice de Malmquist estimado con la metodología DEA. Los resultados obtenidos representan una importante contribución empírica que puede ser utilizada como herramienta de diagnóstico para la formulación de políticas públicas destinadas a mejorar la productividad de fincas lecheras $y$, en general, constituye un aporte al estudio de productividad agraria en países en vías de desarrollo. Se escogió el índice de Malmquist para estimar el cambio en la productividad total de los factores por ser una propuesta no paramétrica que presenta ventajas frente a otras técnicas $y$, también, por su creciente utilización en el campo de las investigaciones agrarias.

\section{Materiales y métodos}

\section{El Índice de Productividad de Malmquist}

Los índices de productividad basados en números índices como el índice de Fisher y los índices de Tornqvist se emplean con frecuencia para medir el crecimiento de la productividad total de los factores (PTF por sus siglas en inglés) (Coelli et al., 2005; Gregg y Rolfe, 2011) y reflejan el desempeño de la industria en su conjunto. Sin embargo, estos ín- 
dices no necesariamente proporcionan información para identificar los factores que contribuyen al crecimiento de la PTF (Coelli et al., 2005).

Otra limitación de los métodos basados en números índices es su incapacidad para diferenciar los cambios en productividad debidos a avances tecnológicos de aquellos que resultan de cambios en la eficiencia y, por tanto, no permiten establecer las causas que originan las diferencias entre la productividad y la eficiencia (Kompas y Che, 2004; Pastor, 1995). El reconocimiento de las limitaciones de los índices tradicionales para estimar la PTF ha orientado el desarrollo de técnicas que descomponen la productividad (O'Donnell, 2009 y 2010). Por estas razones en la presente investigación se descartan los índices tradicionales para estimar la PTF y se recurre al índice de productividad de Malmquist (1953) o IPM, el cual ha sido ampliamente abordado en un contexto no paramétrico (Färe et al., 1998; CoeIli et al., 1998; Tone, 2004). En este índice, que mide el crecimiento de la productividad total de los factores (PTF) de una finca, se cuantifican dos aspectos importantes: a) la mejora en la eficiencia técnica (desplazamiento a la frontera de producción, si la finca era ineficiente al inicio del periodo de evaluación) y b) el progreso o cambio tecnológico evidenciado por el desplazamiento de la frontera tecnológica en el tiempo (las fincas consideradas eficientes al inicio del periodo de estudio aumentan sus niveles de productividad en el tiempo), en un sistema productivo caracterizado por múltiples insumos y productos.

El índice de la variación de productividad Malmquist fue introducido por Caves et al. (1982), inspirados en el trabajo de Malmquist (1953), y su objetivo es medir la variación de la productividad entre dos periodos de tiempo diferentes, utilizando como medida de los cambios en la productividad total la relación (razón o cociente) entre dos funciones distancia. Según estos autores, con el IPM se pueden comparar, en dos momentos diferentes, los niveles de insumos (o inputs), productos (u outputs) y productividad de una misma unidad de decisión (finca), o también, de dos fincas diferentes en el mismo momento o en diferentes momentos.

Para analizar los cambios ocurridos entre esos dos periodos de tiempo, se pueden tener dos tecnologías de producción para realizar la comparación, que corresponden a la del periodo inicial y a la del periodo final, y según cuál de esas tecnologías se asuma como referencia se pueden estimar dos índices de productividad. Esta situación introduce un elemento de arbitrariedad al tener que elegir el periodo de referencia en $t$ o en $t+1$. Färe et $a l$. (1992) construyeron un índice Malmquist basado en DEA, el cual corresponde a la media geométrica de dichos índices.

A diferencia de otras aproximaciones propuestas para medir la productividad, el IPM también proporciona información sobre el origen del cambio de productividad mediante la descomposición de este índice en dos componentes: uno de cambio tecnológico (CT) y otro de cambio en la eficiencia (CE), bajo el supuesto de rendimientos constantes a escala. El primero también es conocido como movimiento de frontera, progreso técnico o Frontier-Shift y recoge la variación debida al desplazamiento de la frontera eficiente, por lo que revela los desplazamientos, entre dos periodos de tiempo, generados por incrementos en la productividad de las fincas inicialmente eficientes. Es decir, se relaciona con el conjunto de innovaciones y cambios en las técnicas agrarias que desplazan la frontera de producción obteniéndose, así, un mayor nivel de producto sin variar la cantidad de insumos utilizados, o bien obtener el mismo nivel de producto, utilizando menor cantidad de insumos en el proceso productivo (Martín, 2000).

El segundo componente cambio en la eficiencia también es conocido como convergencia 
a la frontera o catching-up, y refleja la mejora en la eficiencia de una finca en comparación con sus pares eficientes (las fincas con la mejores prácticas agrarias). O dicho de otra manera, este tipo de eficiencia muestra el resultado de la capacidad que tienen las fincas en la gestión de su proceso productivo para incorporar progreso técnico (Martín, 2000). En concreto, este componente mide la parte de la variación de la productividad global de la finca asociada al factor trabajo (las personas): los niveles de aprendizaje y experiencia del personal, la transferencia de la tecnología agraria, los avances en la gestión y organización de los recursos y los planes de incentivos laborales, entre otros, que en esencia representan factores que no están vinculados directamente con el progreso técnico, pero si con la eficacia con que éste se adopta o incorpora.

Cuando se asumen rendimientos variables a escala el cambio en la eficiencia técnica (CE) se desglosa en cambio en la eficiencia técnica pura (CETP) y cambio en la eficiencia de escala (CEE), que se ajusta más a la realidad del mundo empresarial según la propuesta de Färe et al. (1994). El primer tipo de cambio refleja el resultado que se correspondería con la gestión exclusivamente técnica de la empresa independientemente de su tamaño, y el segundo se derivada del tamaño de la finca con relación a la escala óptima (Martín, 2000). Esta descomposición del índice de Malmquist es un punto que ha generado una importante controversia según Coelli et al. (2005), quienes señalaron que el punto crítico principal es que sí existe cambio en la eficiencia en escala, lo cual implica que la verdadera tecnología de producción es con rendimientos variables a escala y no con rendimientos constantes, pero la descomposición propuesta por Färe et al. (1994) refleja los movimientos en la frontera de rendimientos constantes a escala (RCE) y no en la de rendimientos variables a escala (RVE). Previamente, Ray y Desli (1997) al asomar esta inconsistencia, que fue rápidamente replicada por Färe et al. (1997), propusieron una descomposición alternativa basada en RVE que denominaron componente de cambio en escala, que no es equivalente al cambio en la eficiencia en escala. Aunque este método puede presentar ventajas, presenta el inconveniente de tener dificultades computacionales propiciando infactibilidades en algunos cálculos de RVE entre diferentes periodos (Canós et al., 2010).

Para estimar las medidas de distancia del índice de Malmquist hay que elegir una orientación: a) Hacia los insumos (reducción de las cantidades de insumos aplicados mientras se mantienen constantes los niveles de producto) o b) Hacia los productos (aumento de los niveles de producción mientras los niveles de insumos permanecen constantes). Esta imposición tampoco está exenta de controversia (Ferro y Romero, 2011), ya que en ocasiones tiene sentido (como en la orientación a los insumos si se están estudiando sectores regulados con obligación de provisión por parte del oferente), pero en otras se manifiesta como demasiado rígido o inapropiado (un mercado competitivo donde no hay restricciones a expandir la producción o reducir los insumos).

En resumen, el índice de Malmquist, al estar basado en el método DEA presenta además otras ventajas y desventajas. Entre las primeras se tienen (Schuschny, 2007): 1) Permite trabajar con múltiples insumos y productos, medidos en diferentes sistemas de unidades, 2) Al contrario de los métodos de naturaleza econométrica que emplean una aproximación a la frontera de producción estocástica, la estimación del IPM no requiere la asunción de pleno empleo de los factores productivos, 3) No impone el uso de formas funcionales explícitas para la tecnología, y 4) Las fincas son comparadas con una finca ideal, construida a partir del desempeño de fincas "pares" reales, que son productivamente más eficientes, mediante el cálculo 
de una combinación lineal de estas últimas y 5) el IPM se puede descomponer en cambio técnico y cambio en la eficiencia y, por lo tanto, aporta información sobre los efectos relativos de estos factores.

Entre las desventajas o limitaciones se destacan (Schuschny, 2007): 1) Es sensible a errores de medición. Los "outliers" representados por fincas altamente productivas pueden sesgar los resultados al determinar la frontera de referencia. Por el contrario, las fincas muy poco productivas no afectan los resultados, 2) La exclusión de variables relevantes puede dar lugar a la identificación de ineficiencias (espúreas), 3) Se estiman muy bien las eficiencias (o ineficiencias) "relativas", pero no "absolutas" cuyo objetivo sea obtener resultados potenciales o ideales 4) Por ser una técnica no paramétrica no se recomienda para probar hipótesis de investigación, a menos que se utilicen técnicas de remuestreo, como la metodología bootstrap, en el cálculo del IPM (Simar y Wilson, 1999) y 5) Otra de las debilidades achacadas es que no distingue entre "noise" e ineficiencia técnica, como ocurre en las estimaciones obtenidas a través de fronteras estocásticas. Así, cualquier desviación de la frontera sería considerada, en este caso, como ineficiencia. Debido a ello, los shocks externos desfavorables, que afecten el resultado de una economía, serían captados como ineficiencias (al no computar los efectos aleatorios, la metodología DEA podría sobreestimar la medición de aquéllas).

A pesar de sus desventajas el IPM es una herramienta muy poderosa para calcular el crecimiento de la productividad cuando existen ineficiencias en los procesos productivos de las fincas (Grosskopf, 1993). Es importante destacar que la verdadera tecnología de las empresas agrarias es desconocida (Quirós y Picazo, 2001).
Formulación matemática del Índice de Productividad de Malmquist

El IPM de las fincas orientado a producto y tomando como referencia la tecnología del periodo base $s^{t}$ se define como (Caves et al., 1982):

$$
\operatorname{IPM}_{o}^{t}(x, y)=\frac{D_{o}^{t}\left(x^{t+1}, y^{t+1}\right)}{D_{o}^{t}\left(x^{t}, y^{t}\right)}
$$

Que viene a representar el crecimiento de la productividad medido en términos de distancias verticales, tomando como referencia la tecnología del periodo $t$.

De igual manera, el IPM de las fincas orientado a producto y tomando como referencia la tecnología del periodo siguiente $s^{t+1}$ se define como:

$$
\operatorname{IPM}_{o}^{t+1}(x, y)=\frac{D_{o}^{t+1}\left(x^{t+1}, y^{t+1}\right)}{D_{o}^{t+1}\left(x^{t}, y^{t}\right)}
$$

Que viene a representar el crecimiento de la productividad medido en términos de distancias verticales, tomando como referencia la tecnología del periodo $t+1$.

De manera que, el cambio productivo para una finca puede ser definido con relación a la frontera tecnológica, bien del periodo $t$ o del $t+1$. Con el propósito de obviar la arbitrariedad en la elección de la tecnología de referencia, Färe et al. (1989 y 1992) propusieron un IPM basado en la media geométrica de las dos tecnologías de referencia antes descritas.

Así, el cambio productivo experimentado por una finca entre el periodo $t$ y el periodo $t+1$, medido por el índice de productividad total de Malmquist orientado a producto, puede obtenerse a partir de la expresión siguiente:

$\operatorname{IPM}_{O}\left(x^{t+1}, y^{t+1}, x^{t}, y^{t}\right)=\left[\left(\frac{D_{0}^{t}\left(x^{t+1}, y^{t+1}\right)}{D_{0}^{t}\left(x^{t}, y^{t}\right)}\right)\left(\frac{D_{0}^{t+1}\left(x^{t+1}, y^{t+1}\right)}{D_{0}^{t+1}\left(x^{t}, y^{t}\right)}\right)\right]^{1 / 2}$ 
Una de las principales razones de la utilización de este índice de PTF es que se puede desglosar en dos componentes (Färe et al., 1994):

$$
\begin{gathered}
I P M_{O}\left(x^{t+1}, y^{t+1}, x^{t}, y^{t}\right)=\frac{D_{0}^{t+1}\left(x^{t+1}, y^{t+1}\right)}{D_{0}^{t}\left(x^{t}, y^{t}\right)} \\
{\left[\left(\frac{D_{0}^{t}\left(x^{t+1}, y^{t+1}\right)}{D_{0}^{t+1}\left(x^{t+1}, y^{t+1}\right)}\right)\left(\frac{D_{0}^{t}\left(x^{t}, y^{t}\right)}{D_{0}^{t+1}\left(x^{t}, y^{t}\right)}\right)\right]^{1 / 2}}
\end{gathered}
$$

El primer componente (o término) de esta ecuación (antes del corchete) estima el cambio en la eficiencia técnica relativa (CE). Un resultado mayor que la unidad indica un aumento de la eficiencia técnica relativa con respecto al periodo anterior, mientras que un resultado menor que la unidad evidencia una caída de la eficiencia relativa. Por su parte, un valor igual a la unidad muestra una situación estática.

El segundo componente mide el cambio en el progreso tecnológico (CT). Un cambio tecnológico mayor que la unidad indica progreso tecnológico y menor que la unidad evidencia un retroceso tecnológico. Si es igual a la unidad muestra una situación de estancamiento tecnológico (sin cambios).

Es importante destacar que los dos componentes del cambio productivo pueden evolucionar en direcciones contrapuestas, de manera que, por ejemplo, es posible que en una finca ocurran simultáneamente un progreso tecnológico y una desmejora de la eficiencia técnica o viceversa (Maroto, 2007; Coll-Serrano et al., 2009; Karanja et al., 2012), aunque bajo determinadas condiciones, las caídas de productividad pueden ser compatibles con el comportamiento racional de las empresas, sin necesidad de asumir regreso tecnológico (Pastor, 1994). Un caso hipotético de regresión tecnológica podría ser la incorporación de un lote de vacas de ordeño genéticamente muy superiores a las reemplazadas (fuente de progreso tecnológico), pero la combinación de varios factores en esa finca en particular, tales como fallas en el servicio de electricidad e impericia del personal de ordeño (fuentes de ineficiencia relativa) limitan significativamente el potencial de las vacas, hasta el punto de que apenas se supera la productividad promedio de leche por vaca reemplazada del periodo anterior (ligero progreso tecnológico). Estas ineficiencias aumentan la brecha (desmejora de la eficiencia) que ya existía en el periodo anterior entre la finca comparada y las fincas eficientes, que también adquirieron vacas de ordeño con alto potencial genético.

Dado que en el mundo real, las fincas, por lo general, no presentan procesos productivos caracterizados por rendimientos constantes a escala, sino por el contrario, esos procesos están gobernados por las tecnologías de rendimientos variables a escala, es conveniente utilizar la propuesta de Färe et al. (1994), que desglosa la eficiencia técnica relativa en dos componentes: cambio en la eficiencia técnica pura (CETP) y cambio en la eficiencia de escala (CEE), por lo que partiendo del componente de cambio eficiencia técnica relativa la fórmula queda expresada de la forma siguiente:

$$
\begin{aligned}
\frac{D_{0}^{t+1}\left(x^{t+1}, y^{t+1}\right) C R S}{D_{0}^{t}\left(x^{t+1}, y^{t+1}\right) C R S}= & {\left[\frac{D_{0}^{t+1}\left(x^{t+1}, y^{t+1}\right) V R S}{D_{0}^{t}\left(x^{t}, y^{t}\right) V R S}\right] } \\
& {\left[\left(\frac{D_{0}^{t}\left(x^{t}, y^{t}\right) V R S}{D_{0}^{t}\left(x^{t}, y^{t}\right) C R S}\right)\left(\frac{D_{0}^{t+1}\left(x^{t+1}, y^{t+1}\right) C R S}{D_{0}^{t+1}\left(x^{t+1}, y^{t+1}\right) V R S}\right)\right] }
\end{aligned}
$$

El primer término de la ecuación mide los cambios en la eficiencia técnica pura asumiendo rendimientos variables a escala y el segundo término mide los cambios en la productividad que provienen de las modificaciones en la escala de producción de la finca.

Un valor mayor que la unidad en el IPM indica una mejora en la productividad y menor que la unidad evidencia merma de la productividad, con respecto al periodo inicial o 
base. Si es igual a la unidad muestra una situación de estancamiento productivo (sin cambios).

La estimación del IPM requiere del cálculo de las funciones de distancia correspondientes, para lo cual existen varias maneras de abordarlo (Färe et al., 1998). De todas ellas, ha cobrado mucho auge la basada en la resolución de un problema de programación matemática tipo análisis envolvente de datos, donde la función distancia de una finca $k^{\prime}$ en el periodo $t$ es estimada a partir de la resolución de un problema de programación lineal como el que sigue:

$$
\left[D_{\mathbf{0}}^{t}\left(x^{k^{\prime}, t}, y^{k^{\prime}, t}\right)\right]^{-\mathbf{1}}=\operatorname{má} x \theta^{k^{-}}
$$

Donde $x$ y $y$ representan, respectivamente, los insumos y productos del proceso productivo agrario que realiza la finca $k^{\prime}$ y $k=1, \ldots K$ fincas que componen la muestra contra las cuales se compara su resultado.

De manera que:

$x=$ número de vacas totales y los gastos totales en unidades monetarias

$y=$ producción anual de leche expresada en litros y el número de los nacimientos de becerros por año, que genera ese proceso productivo

s.a

$$
\begin{aligned}
& \sum_{k=1}^{K} \lambda^{k, t} y_{m}^{k, t} \geq \theta^{k^{\prime}} y_{m}^{k, t} \quad m=1, \ldots . ., M \\
& \sum_{k=1}^{K} \lambda^{k, t} x^{k, t} \leq x_{n}^{k, t} \quad n=1, \ldots ., N \\
& \lambda^{k, t} \geq 0 \quad k=1, \ldots ., K
\end{aligned}
$$

Similar es la expresión matemática que sirve para calcular la función de distancia con respecto del periodo $t+1$, para lo cual se sustituye el superíndice $t$ por $t+1$ en las ecuaciones antes presentadas. Con respecto a la función distancia $D_{0}^{t}\left(x^{t+1}, y^{t+1}\right)$ se estima de forma simi- lar, con la condición de sustituir el superíndice $t$ por $t+1$ en el lado derecho de las restricciones. Asimismo, la estimación de la función $D_{0}{ }^{t+1}\left(x^{t}, y^{t}\right)$ se concreta reemplazando el superíndice $t$ por $t+1$ en el lado izquierdo de las restricciones establecidas para el modelo.

Las distancias respecto a la tecnología con rendimientos variables a escala se obtienen imponiendo la restricción adicional de que la suma de los elementos del vector de intensidades debe ser igual a la unidad (Afriat, 1972; Banker et al., 1984).

Para la estimación del IPM en la presente investigación se empleó el programa informático Win4deap versión 2.1 (Coelli, 1996), con base a un modelo orientado a producto, asumiendo rendimientos variables a escala.

\section{Datos y muestra}

El estudio abarcó el período 2006 - 2008 y se realizó en la Empresa Pecuaria Genética Valle Del Perú en el municipio San José de las Lajas, Cuba, la cual dispone de más de 13.542 hectáreas destinadas a la ganadería. La muestra estuvo conformada inicialmente por 30 fincas, que representan el $56 \%$ del total de fincas. Los datos se obtuvieron por medio de diagnósticos efectuados en la totalidad de las unidades de la empresa, pero solo se incluyeron estas explotaciones que dispusieron de información completa. Posteriormente, se eliminaron seis fincas por considerarse que sus valores eran un tanto atípicos y, por ello, la muestra final fue de 24 explotaciones lecheras, a cada una de las cuales se les midieron cuatro variables (dos insumos y dos productos). Esta información fue recabada con anterioridad para un estudio de caracterización de las fincas, lo cual impuso algunas restricciones para el presente análisis.

En la totalidad de las unidades se practica el doble ordeño (predominantemente meca- 
nizado) y disponen de equipos de refrigeración para conservar la leche. En la gran mayoría de fincas se pastoreó a los animales solamente 9 horas diurnas. El genotipo predominante es el mestizo Siboney, seguido del Siboney puro. Otras características de las fincas en valores promedios son: el área agrícola por finca es de 81,65 ha, se explotan 77,0 vacas por finca, lo que da una carga de 0,95 vacas/ha y un área de pastos cultivados de $35,4 \%$.

Los insumos correspondieron al número de vacas totales y a los gastos totales (unidades monetarias nominales) del proceso productivo medidos en una dimensión anual, y como productos se consideraron la producción anual de leche expresada en litros y el número de nacimientos de becerros por año. Los gastos totales nominales fueron deflactados utilizando el índice de precios para la agricultura, ganadería y silvicultura (Oficina Nacional de Estadísticas de Cuba, 2012), tal como lo hicieron Lerdon et al. (2010), Mkhabela (2011) y Li et al., (2010). La producción de leche, así como los insumos, gastos totales y número de vacas totales ya han sido empleados en estudios sobre la eficiencia en fincas lecheras, solo que las dos primeras variables fueron expresados con relación a un día (Arzubi y Berbel, 2002).

Para analizar el crecimiento de la productividad de las fincas se compararon los periodos 2006-2007, 2007-2008 y 2006-2008.

Las unidades estudiadas son explotaciones de tamaño pequeño, según la clasificación de Lerdon et al. (2010), con una producción láctea anual de 54.243,5 l/fincas y un promedio de 77,6 vacas por finca, y con una gran variabilidad en cuanto a producción y utilización de insumos por parte de las fincas.

\section{Resultados y discusión}

En una primera instancia se analiza el periodo comprendido entre los años 2006 y
2007. Al indagar en la columna (5) de la Tabla 1, se observa que la finca número 16 fue la que experimentó el mayor incremento de la productividad total (IPM =1,324), al lograr $32,4 \%[(1,324-1) * 100]$, que se debió únicamente a la mejora de su eficiencia técnica (CE en la columna 1$)$ en $35,1 \%$ [(1,351-1)*100], ya que experimentó un pequeño retroceso tecnológico (CT en la columna 2 ) de $2,0 \%$. El valor del CE indica que, de todas las fincas ineficientes, ésta fue la que se acercó más al nivel de producción de sus Benchmarks en el año 2007 en comparación con una mayor distancia que presentaba en el año anterior. El valor del CT revela una ligera caída de la tasa de conversión producto-insumo (disminuyó la producción de leche y aumentaron las vacas totales).

La mejora de la eficiencia (CE) se debió exclusivamente a un mejor uso de la tecnología disponible (CETP = 1,367; que equivale a un incremento del $36,7 \%)$, debido a que presentó una ligera desmejora en la eficiencia de escala (EE) en $1,2 \%$, al operar la finca a un tamaño menos óptimo en comparación con el año 2006.

Por otra parte, la finca número 18 sufrió la mayor caída (decremento) de la productividad, al reflejar un IPM de 0,710 , que equivale a un descenso de $29,0 \%$ [(1-0,710)*100], que se debió más a la desmejora de la eficiencia $(26,9 \%)$, que al retroceso tecnológico $(2,9 \%)$. La merma de la eficiencia técnica no fue importante por el hecho de operar a una escala subóptima $(2,1 \%)$, pero sí por el uso inadecuado (por parte del recurso humano de la finca) de la tecnología disponible (25,3\%), por lo cual esta finca, para el segundo año considerado, se alejó de la frontera de producción que fijaron sus benchmarks o fincas eficientes (considerando rendimientos variables a escala), así como del segmento de dicha frontera donde se opera a la escala apropiada. De manera que las fincas ubicadas en la frontera de producción de rendimientos variables a escala son las que alcanzan las máximas tasas de conversión de insumos en 
Tabla 1. Descomposición del índice de productividad de Malmquist de 24 unidades lecheras de una región de Cuba (periodo 2006-2007)

Table 1. Decomposition of the Malmquist productivity index of 24 dairy units of a region of Cuba (period 2006-2007)

\begin{tabular}{|c|c|c|c|c|c|c|c|c|c|c|c|}
\hline $\mathrm{N}^{\circ}$ & $\begin{array}{l}\text { CE } \\
(1)\end{array}$ & $\begin{array}{l}C T \\
(2)\end{array}$ & $\begin{array}{l}\text { ETP } \\
\text { (3) }\end{array}$ & $\begin{array}{l}\mathrm{EE} \\
\text { (4) }\end{array}$ & $\begin{array}{l}\text { IPM } \\
(5)\end{array}$ & $\mathrm{N}^{\circ}$ & $\begin{array}{l}\text { CE } \\
(1)\end{array}$ & $\begin{array}{l}C T \\
(2)\end{array}$ & $\begin{array}{l}\text { CETP } \\
(3)\end{array}$ & $\begin{array}{l}\text { CEE } \\
(4)\end{array}$ & $\begin{array}{l}\text { IPM } \\
(5)\end{array}$ \\
\hline 1 & 1,000 & 1,032 & 1,000 & 1,000 & 1,032 & 13 & 0,905 & 0,959 & 0,929 & 0,974 & 0,868 \\
\hline 2 & 0,762 & 0,947 & 0,945 & 0,806 & 0,722 & 14 & 1,227 & 1,034 & 1,529 & 0,803 & 1,269 \\
\hline 3 & 0,887 & 0,918 & 1,000 & 0,887 & 0,814 & 15 & 0,820 & 1,046 & 0,837 & 0,980 & 0,858 \\
\hline 4 & 1,094 & 1,026 & 1,077 & 1,016 & 1,123 & 16 & 1,351 & 0,980 & 1,367 & 0,988 & 1,324 \\
\hline 5 & 0,939 & 1,042 & 0,941 & 0,998 & 0,979 & 17 & 1,142 & 0,996 & 1,211 & 0,943 & 1,137 \\
\hline 6 & 0,940 & 1,002 & 0,998 & 0,942 & 0,941 & 18 & 0,731 & 0,971 & 0,747 & 0,979 & 0,710 \\
\hline 7 & 1,159 & 0,872 & 1,140 & 1,016 & 1,011 & 19 & 0,950 & 0,986 & 0,944 & 1,006 & 0,937 \\
\hline 8 & 1,049 & 0,998 & 1,035 & 1,013 & 1,046 & 20 & 0,848 & 1,007 & 1,000 & 0,848 & 0,854 \\
\hline 9 & 1,110 & 1,002 & 1,020 & 1,088 & 1,112 & 21 & 1,023 & 0,994 & 1,000 & 1,023 & 1,018 \\
\hline 10 & 1,295 & 0,976 & 1,172 & 1,105 & 1,264 & 22 & 0,980 & 0,989 & 0,934 & 1,049 & 0,970 \\
\hline 11 & 1,032 & 0,997 & 1,032 & 1,000 & 1,029 & 23 & 0,844 & 0,959 & 1,000 & 0,844 & 0,809 \\
\hline 12 & 0,944 & 1,000 & 0,944 & 1,000 & 0,944 & 24 & 1,234 & 0,976 & 1,346 & 0,917 & 1,204 \\
\hline \multicolumn{7}{|c|}{ Media geométrica } & 0,998 & 0,987 & 1,035 & 0,964 & 0,986 \\
\hline
\end{tabular}

CE: Cambio en la eficiencia técnica CT: Cambio tecnológico IPM: Índice de Productividad de Malmquist CETP: Cambio en la eficiencia técnica pura CEE: Cambio en la eficiencia de escala.

CE: Change in technical efficiency CT: Technological change IPM: Malmquist Productivity Index CETP: Change in pure technical efficiency CEE: Change in scale efficiency.

productos con la tecnología disponible (asumiendo un alto nivel de homogeneidad de las tecnologías de las fincas comparadas). Por ello, las estrategias más idóneas de las fincas ineficientes (no utilizan apropiadamente la tecnología disponible) se enmarcan en un proceso de benchmarking (Camp, 2001), que consiste en imitar (en el contenido y en la ejecución) las mejores prácticas (asociadas con el know-how) de las fincas benchmark (por ejemplo las relacionadas con los programas de salud y alimentación animal, y capacitación del personal, entre otras). Para mejorar la eficiencia de escala, la finca tam- bién debe ubicarse (o acercarse) en el segmento de rendimientos constantes de la frontera de producción.

En el mismo orden de ideas, destaca una importante heterogeneidad en lo que se refiere a cambios de productividad dentro del grupo de fincas estudiado, lo cual también ha sido reportado por Jan (2011), quien precisa que se requiere más investigación para determinar las causas de esa heterogeneidad.

Considerando la media geométrica de la muestra de fincas se puede afirmar que, para este periodo (años 2006 al 2007), hubo una pe- 
queña desmejora en la productividad $(1,4 \%)$, debido a una ligera caída de la eficiencia técnica $(0,2 \%)$ y a un pequeño decremento tecnológico (1,3\%). La desmejora en la eficiencia técnica se debió a que un mejor uso de la tecnología disponible $(3,5 \%)$ fue apenas superado por la disminución de la eficiencia de escala $(3,6 \%)$. En general, la mitad de la fincas de la muestra mantuvo o incrementó sus niveles de productividad en este periodo.

En el segundo periodo considerado (años 2007 al 2008), la finca número 21 fue la que experimentó el mayor incremento de la productividad (Tabla 2), al lograr un 36,4\%, que se de- bió totalmente, a la mejora de la eficiencia técnica $(37,2 \%)$, ya que presentó un ligero regreso tecnológico $(0,5 \%)$. La mejora de la eficiencia técnica se explica exclusivamente porque esta finca se movió hacia una escala (o tamaño) de producción más apropiado (37,2\%), pues el nivel de uso de la tecnología (CETP = $1,00)$ fue el mismo del año base de comparación (año 2007). El mejoramiento de su escala de producción se puede explicar mediante el comportamiento (diferencias aritméticas) de las tasas de variación anual (periodos 20062007 y 2007-2008), de manera que esta finca aumentó, con respecto a las tasas del periodo anterior, los outputs producción de leche en

Tabla 2. Descomposición del índice de productividad de Malmquist de 24 unidades lecheras de una región de Cuba (periodo 2007-2008)

Table 2. Decomposition of the Malmquist productivity index of 24 dairy units of a region of Cuba (period 2007-2008)

\begin{tabular}{|c|c|c|c|c|c|c|c|c|c|c|c|}
\hline $\mathrm{N}^{\circ}$ & CE & $\mathrm{CT}$ & CETP & CEE & IPM & $\mathrm{N}^{\circ}$ & CE & CT & CETP & CEE & IPM \\
\hline 1 & 1,000 & 0,906 & 1,000 & 1,000 & 0,906 & 13 & 1,020 & 1,011 & 1,062 & 0,961 & 1,031 \\
\hline 2 & 0,997 & 0,970 & 0,907 & 1,099 & 0,968 & 14 & 1,229 & 0,941 & 0,988 & 1,243 & 1,156 \\
\hline 3 & 1,128 & 1,023 & 1,000 & 1,128 & 1,154 & 15 & 0,708 & 0,917 & 0,638 & 1,110 & 0,649 \\
\hline 4 & 1,309 & 0,937 & 1,025 & 1,277 & 1,226 & 16 & 0,809 & 1,020 & 0,837 & 0,967 & 0,825 \\
\hline 5 & 0,845 & 0,949 & 1,165 & 0,726 & 0,802 & 17 & 1,142 & 0,973 & 1,152 & 0,991 & 1,111 \\
\hline 6 & 0,957 & 0,985 & 1,002 & 0,955 & 0,942 & 18 & 1,152 & 0,999 & 1,165 & 0,989 & 1,151 \\
\hline 7 & 0,985 & 1,016 & 0,933 & 1,055 & 1,000 & 19 & 0,994 & 1,004 & 1,025 & 0,969 & 0,997 \\
\hline 8 & 1,000 & 1,000 & 1,000 & 1,000 & 1,000 & 20 & 1,144 & 0,989 & 0,788 & 1,452 & 1,132 \\
\hline 9 & 1,085 & 0,978 & 1,079 & 1,006 & 1,061 & 21 & 1,372 & 0,995 & 1,000 & 1,372 & 1,364 \\
\hline 10 & 1,082 & 0,970 & 1,105 & 0,979 & 1,049 & 22 & 1,090 & 0,996 & 0,981 & 1,111 & 1,086 \\
\hline 11 & 0,957 & 0,993 & 1,000 & 0,957 & 0,949 & 23 & 0,746 & 1,031 & 0,701 & 1,065 & 0,769 \\
\hline 12 & 1,191 & 0,952 & 1,184 & 1,005 & 1,134 & 24 & 1,008 & 1,000 & 0,914 & 1,103 & 1,008 \\
\hline \multicolumn{7}{|c|}{ Media geométrica } & 1,027 & 0,981 & 0,975 & 1,053 & 1,007 \\
\hline
\end{tabular}

CE: Cambio en la eficiencia técnica CT: Cambio tecnológico IPM: Índice de Productividad de Malmquist CETP: Cambio en la eficiencia técnica pura CEE: Cambio en la eficiencia de escala.

CE: Change in technical efficiency CT: Technological change IPM: Malmquist Productivity Index CETP: Change in pure technical efficiency CEE: Change in scale efficiency. 
$5 \%$ y nacimientos de becerros en $15 \%$, y aunque los insumos gastos totales crecieron en $4 \%$ (por debajo de las tasas de los productos), las vacas totales se redujeron en un $34 \%$ (del 2006 al 2007 aumento en $16 \%$ y del 2007 al 2008 se redujeron en $18 \%$ ). De esta forma la finca mejoró en el periodo 2007-08 su estructura producto-insumo, lo cual le permitió acercarse a la zona media de la frontera de producción donde operan las fincas con el tamaño más apropiado.

Por otra parte, la finca número 15 sufrió la mayor caída (decremento) de la productividad, al reflejar un descenso de $35,1 \%$ de la productividad, que se debió más a la desmejora de la eficiencia técnica $(29,2 \%)$ que al regreso tecnológico de $8,3 \%$. La caída de la eficiencia se debió a que la eficiencia técnica pura disminuyó significativamente en $36,2 \%$ (CETP $=0,638)$, no pudiendo compensar la mejora de eficiencia $(11,0 \%)$ proveniente de la operación a una escala más adecuada. Debido a que se empleó un modelo de optimización orientado a producto, lo más conveniente para esta finca (en general para las que presenten problemas de productividad) es elevar significativamente la producción de leche y los nacimientos de becerros, con los mismos niveles (o valores muy cercanos) de insumos aplicados al final del periodo evaluado. Obviamente también se pueden reducir insumos manteniendo los actuales niveles de producción, pero esta opción no es muy compatible con el principio de seguridad y soberanía agroalimentaria, que rige para el sector agropecuario, y que es particularmente importante en momentos en que se manifiestan significativos déficits en la producción de alimentos. Para el proceso de mejoramiento de su productividad, los dueños de esta finca pueden guiarse por las que tienen una estructura de insumos similar y que son ciento por ciento eficientes (asumiendo preferiblemente la frontera de rendimientos variables a escala), por lo cual se consideran las fincas líderes (pares eficientes) que son detectadas utilizando el método DEA para estimar la eficiencia (Toro et al., 2010). Para este caso específico de la finca 5, sus pares eficientes en orden de importancia son las fincas 1, 11, 3 y 21.

En general, el $62,5 \%$ de las fincas objeto de estudio incrementó sus niveles de productividad en este periodo.

Considerando los valores promedios de la muestra de fincas, se puede afirmar que, para este periodo, el pequeño aumento en la productividad $(0,7 \%)$, se debió únicamente a ligeros incrementos de la eficiencia técnica $(2,7 \%)$, que superó el regreso tecnológico experimentado $(1,9 \%)$. La eficiencia técnica mejoró debido a que se operó a una escala de producción más adecuada $(5,3 \%)$, pero el uso inapropiado de la tecnología disponible (el CETP disminuyó en 2,5\%) canceló en gran parte la ventaja competitiva obtenida.

Al comparar los cambios en la productividad y sus componentes en las unidades lecheras durante los periodos 2006 al 2007 y 2007 al 2008 (Tabla 3), destaca que la caída de la productividad total de 1,4\% ocurrida en el primer periodo no fue totalmente contrarrestada por un ligero aumento en el segundo periodo considerado $(0,7 \%)$, por lo cual, en promedio, hubo una pequeña caída de la productividad (aprox. 0,4\%) en los tres años comparados. Al indagar en las causas de este resultado mediante el examen de sus componentes, se evidencia que hubo mejora (aunque muy pequeña) en el segundo periodo con respecto al primero en cambio de eficiencia técnica, que contribuyó a que el promedio fuese positivo de 1,2\%. El regreso tecnológico se acentúo ligeramente en el segundo periodo con respecto al primero, por lo cual el promedio indica un ligero retroceso tecnológico de 1,6\%. El cambio favorable en la eficiencia se debió a una ligera mejora en cuanto a la escala de producción $(0,8 \%)$ y en el uso de la tecnología disponible $(0,5 \%)$. Estos valores promedios son los que caracterizan la productividad del sistema estudiado. 
Tabla 3. Comparación de los cambios en la productividad y sus componentes en 24 unidades lecheras (periodos 2006-2007 y 2007-2008)

Table 3. Comparison of changes in productivity and its components in 24 dairy units (periods 2006-2007 and 2007-2008)

\begin{tabular}{lllllll}
\hline Periodos & CE & CT & CETP & CEE & IPM & IPM (\%) \\
\hline 2006-2007 & 0,998 & 0,987 & 1,035 & 0,964 & 0,986 & $-1,40$ \\
2007-2008 & 1,027 & 0,981 & 0,975 & 1,053 & 1,007 & 0,70 \\
\hline Media geométrica & 1,012 & 0,984 & 1,005 & 1,008 & 0,996 & $-0,36$ \\
\hline Tasa de crecimiento anual $(\%)^{(1)}$ & 1,43 & $-0,30$ & $-2,99$ & 4,42 & 1,05 & \\
\hline
\end{tabular}

CE: Cambio en la eficiencia técnica CT: Cambio tecnológico IPM: Índice de Productividad de Malmquist CETP: Cambio en la eficiencia técnica pura CEE: Cambio en la eficiencia de escala.

(1) Se empleó la ecuación: $x=x_{0} \mathrm{e}^{g t} \rightarrow \ln x=\ln x+g t$

CE: Change in technical efficiency CT: Technological change IPM: Malmquist Productivity Index CETP: Change in pure technical efficiency CEE: Change in scale efficiency.

(1) Equation used: $x=x_{0} e^{g t} \rightarrow \ln x=\ln x+g t$

Para corroborar las variaciones señaladas en el párrafo anterior, se realizó un análisis longitudinal (cross time) del comportamiento de las tasas anuales del IPM y sus componentes del primer periodo respecto al segundo. Destaca que la productividad se incrementó a una tasa de 1,05\%, influida únicamente por la tasa de $1,43 \%$ de cambio en la eficiencia, ya que se evidenció una tasa de regreso tecnológico de 0,3\%. En el primer componente resalta la caída de la eficiencia técnica pura a una tasa de 2,99\%, contrarrestada por una tasa positiva del cambio en la eficiencia de escala $(4,42 \%)$.

Finalmente, en la Tabla 4 se presenta un resumen de los valores promedios de los cambios en la productividad y sus componentes en 24 unidades lecheras considerando los tres años de estudio, estimados por la media geométrica de los periodo 2006-07 y 2007-08, que representa la media anual de los índices. Desde esta perspectiva, la finca número 14 fue la que experimentó el mayor incremento anual de la productividad total $(21,1 \%)$, que se debió únicamente a la mejora de su eficiencia técnica en
$22,8 \%$, ya que mostró un retroceso tecnológico de 1,4\%. Al profundizar en el análisis destaca que la mejora de su eficiencia por un uso más apropiado de la tecnología disponible $(22,9 \%)$ superó con creces el ligero descenso de su eficiencia de escala $(0,1 \%)$.

Por otra parte, la finca número 15 sufrió la mayor caída (decremento) de la productividad total $(25,4 \%)$, que se debió más a la desmejora de la eficiencia $(23,8 \%)$ que al retroceso tecnológico $(2,0 \%)$. Aunque mejoró al operar a una escala más apropiada (4,3\%), el uso inadecuado de la tecnología disponible es el factor crítico en esta finca, al abatir su eficiencia en $27,0 \%$, alejándola de sus competidoras eficientes. En general, el $50,0 \%$ de las fincas mantuvo o incrementó sus niveles de productividad en este periodo; solo en una finca (que representa el $4,2 \%$ del total) hubo progreso tecnológico y de un $58,3 \%$ que mantuvo o elevó su eficiencia técnica, el $66,7 \%$ mantuvo o elevó su ETP y el 54,2\% mejoró su tamaño de operación. Por otra parte, ninguna finca logró progresar simultáneamente en productividad y todos sus componentes. 
Tabla 4. Descomposición del índice de productividad de Malmquist de 24 unidades lecheras de una región de Cuba (2006-2008)

Table 4. Decomposition of the Malmquist productivity index of 24 dairy units of a region of Cuba (2006-2008)

\begin{tabular}{rccccccccccc}
\hline $\mathrm{N}^{\circ}$ & CE & CT & CETP & CEE & IPM & $\mathrm{N}^{\circ}$ & CE & CT & CETP & CEE & IPM \\
\hline 1 & 1,000 & 0,967 & 1,000 & 1,000 & 0,967 & 13 & 0,961 & 0,984 & 0,993 & 0,967 & 0,946 \\
2 & 0,872 & 0,959 & 0,926 & 0,941 & 0,836 & 14 & 1,228 & 0,986 & 1,229 & 0,999 & 1,211 \\
3 & 1,000 & 0,969 & 1,000 & 1,000 & 0,969 & 15 & 0,762 & 0,980 & 0,730 & 1,043 & 0,746 \\
4 & 1,197 & 0,981 & 1,051 & 1,139 & 1,174 & 16 & 1,045 & 1,000 & 1,070 & 0,977 & 1,045 \\
5 & 0,891 & 0,995 & 1,047 & 0,851 & 0,886 & 17 & 1,142 & 0,985 & 1,181 & 0,967 & 1,124 \\
6 & 0,948 & 0,993 & 1,000 & 0,948 & 0,942 & 18 & 0,918 & 0,985 & 0,932 & 0,984 & 0,904 \\
7 & 1,068 & 0,941 & 1,031 & 1,036 & 1,005 & 19 & 0,971 & 0,995 & 0,984 & 0,988 & 0,966 \\
8 & 1,024 & 0,999 & 1,017 & 1,007 & 1,023 & 20 & 0,985 & 0,998 & 0,888 & 1,110 & 0,983 \\
9 & 1,097 & 0,990 & 1,049 & 1,046 & 1,087 & 21 & 1,185 & 0,994 & 1,000 & 1,185 & 1,178 \\
10 & 1,184 & 0,973 & 1,138 & 1,040 & 1,152 & 22 & 1,033 & 0,993 & 0,957 & 1,080 & 1,026 \\
11 & 0,994 & 0,995 & 1,016 & 0,978 & 0,988 & 23 & 0,793 & 0,994 & 0,837 & 0,948 & 0,789 \\
12 & 1,060 & 0,976 & 1,057 & 1,003 & 1,035 & 24 & 1,115 & 0,988 & 1,109 & 1,006 & 1,102 \\
\hline
\end{tabular}

CE: Cambio en la eficiencia técnica CT: Cambio tecnológico IPM: Índice de Productividad de Malmquist CETP: Cambio en la eficiencia técnica pura CEE: Cambio en la eficiencia de escala.

CE: Change in technical efficiency CT: Technological change IPM: Malmquist Productivity Index CETP: Change in pure technical efficiency CEE: Change in scale efficiency.

Al comparar los resultados de la presente investigación con los reportados por la literatura especializada de otros países, destaca una importante variabilidad del índice de productividad y sus componentes. Por ejemplo, Graham (2009) mediante el índice de Malmquist (IPM), calculado con el método DEA, estimó el crecimiento promedio de productividad para una muestra de 22 fincas en $12,5 \%$ anual. El estudio reportó que una disminución del 5,8\% en la eficiencia técnica fue contrarrestada por un incremento de 19,5\% en el progreso tecnológico. Estas variaciones son muy grandes en comparación con los resultados obtenidos en la presente in- vestigación y, también, con respecto a la mayoría de los cambios de productividad reportados en fincas lecheras, que tienden a estar entre 0 y 2\% (Gray et al., 2011; Jan, 2011; Karanja et al., 2012). Es probable que estas mediciones estén afectadas por factores climáticos coyunturales, los cuales pueden incidir de manera significativa en las estimaciones de productividad obtenidas de datos de un pequeño número de fincas, estudiadas por un corto periodo de tiempo. Es importante señalar que el IPM también puede ser influenciado por otros factores, tales como los tipos de productos e insumos empleados en sus cálculos, la calidad de la información 
colectada (Luik et al., 2011), el periodo analizado, así como por el nivel de desarrollo del país donde se realiza el estudio (Jan, 2011), por lo cual, las comparaciones entre grupos de fincas de países o regiones diferentes deben ser tomadas con la debida precaución.

Luik et al. (2011) también reportaron cambios del IPM con algunos valores interanuales de crecimiento o decrecimiento rondando un $20,0 \%$, en una muestra de 58 explotaciones lecheras de Estonia analizadas durante el período 2001-2009, mostrando además, que el progreso tecnológico, más que la eficiencia técnica, fue el factor de mayor incidencia en la productividad.

En el mismo orden de ideas, los resultados globales que se obtuvieron en la presente investigación son similares a los reportados por Karanja et al. (2012) en el estado de Victoria en Australia, donde el análisis global para un periodo de tres años, en una muestra de 35 fincas, mostró un estancamiento de la productividad total de los factores, debido a que el pequeño aumento del cambio en la eficiencia técnica $(1,4 \%)$ fue contrarrestado por un regreso tecnológico de la misma proporción. Este atípico o inusual regreso tecnológico es teóricamente factible (Maroto 2007; Coll-Serrano et al., 2009) y, en el caso de las fincas de Victoria, fue explicado por factores externos que influyeron negativamente en la tecnología que determina la frontera de producción, tales como los problemas de medición de datos, las variaciones climáticas, la degradación ambiental, los cambios en las restricciones regulatorias y la insuficiente investigación y políticas de desarrollo. Sin embargo, estimaron que la disponibilidad de agua de riego y los factores climáticos (en particular en la Región Norte) fueron probablemente las causas primarias.

Asimismo, en una serie de estudios previos de productividad total de los factores en las explotaciones lecheras también se reportó el pro- blema de la regresión tecnológica. Así, Moreira-López et al. (2006) encontraron una significativa regresión tecnológica promedio de $17 \%$ anual en 46 explotaciones lecheras en Argentina, al analizar los periodos de 1997-98 a 2001-02. Tauer (1998) observó que 17 de las 70 granjas lecheras de Nueva York experimentaron una regresión tecnológica. Brummer et al. (2002) reportaron un 9\% de regresión tecnológica para las granjas lecheras polacas, mientras Latruffe y Fogarasi (2009) reportaron una regresión tecnológica anual del $14 \%$ en el caso de Hungría. Finalmente, Singh y Singh (2012) reportaron resultados similares al estudiar la productividad agrícola (a nivel agregado) de la India, donde el aumento de la eficiencia técnica fue contrarrestado por un retroceso tecnológico debido, entre otras razones, a la caída de las inversiones públicas en investigación y desarrollo.

Para indagar sobre las posibles causas del retroceso tecnológico en las fincas cubanas objeto de estudio, es menester explicar el comportamiento de los productos e insumos durante el periodo 2006-2008 mostrados en la Figura 1, donde la variable producción de leche por finca, a diferencia de las demás, fue graficada con referencia en la ordenada (eje) de la derecha. Allí se observa que los dos productos (Producción de leche y nacimientos) tendieron a crecer durante el periodo, al igual que gastos totales, pero el insumo vacas totales aumentó en el 2007, para luego decrecer ligeramente. Este aumento de las vacas no se reflejó proporcionalmente en la producción de leche y en los nacimientos de becerros de ese año, lo cual provocó una importante caída de la productividad asociada a este insumo, hecho que se corrobora en la Figura 2, donde se presenta la evolución de la productividad de las fincas en el periodo 2006-2008. La producción de leche por vaca fue graficada con referencia a la ordenada (eje) de la derecha, y se estimó el número de nacimientos de becerros por cada 1.000 uni- 


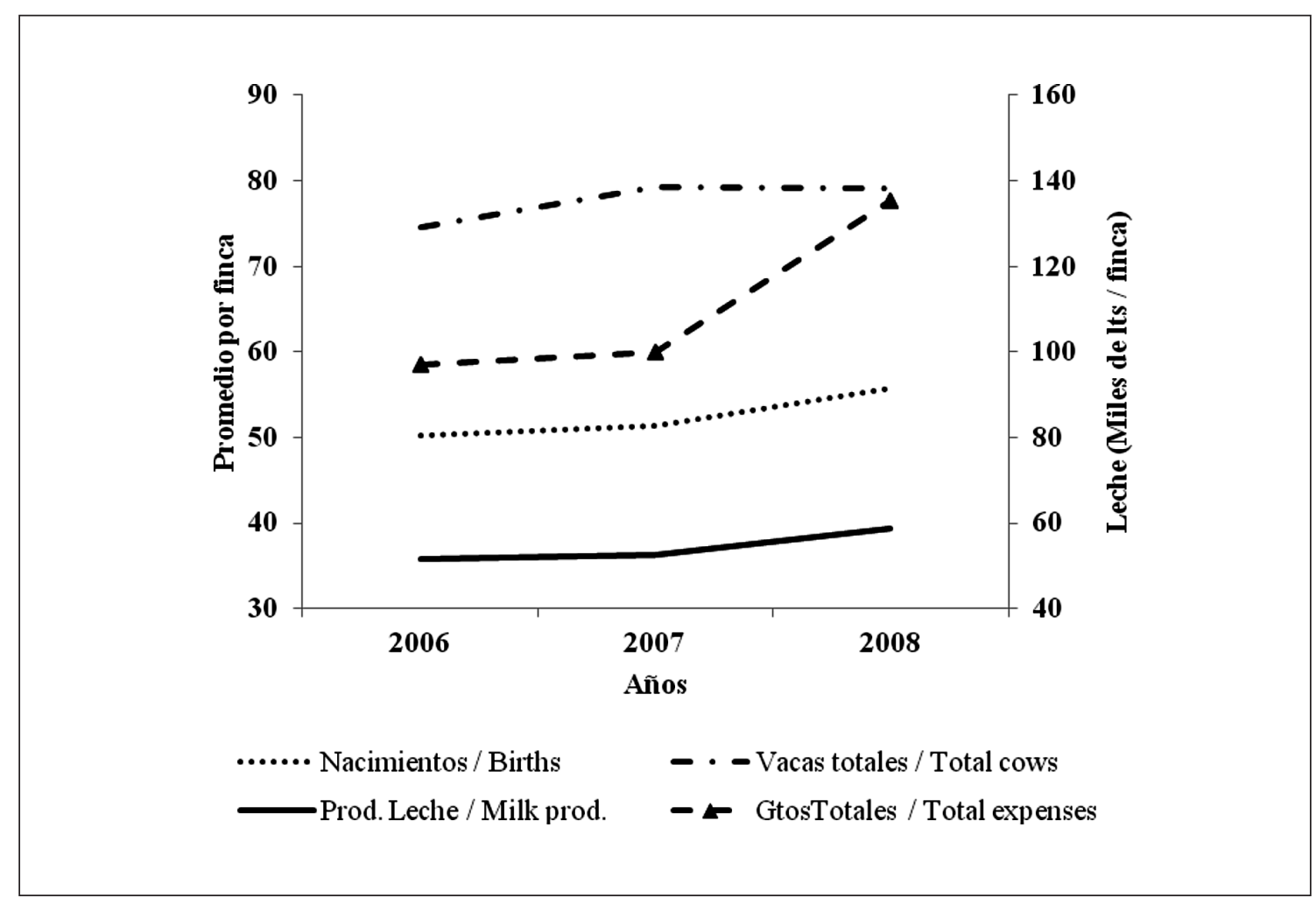

Figura 1. Comportamiento de los productos e insumos de 24 explotaciones lecheras durante el periodo 2006-2008.

Figure 1. Behavior of the outputs and inputs of 24 dairy farms in 2006-2008.

dades monetarias de gastos. Según este gráfico, los nacimientos y los litros de leche por vaca, decrecieron en el año 2007, para incrementarse al año siguiente, lo cual compensó la caída de los rendimientos de estos mismos productos, con referencia a los gastos totales en el 2008. Así pues, el aumento de las vacas totales en el 2007 abatió la relación de conversión producto-insumo, afectando a todas las fincas, incluyendo a las que estaban en la frontera de producción (fincas eficientes), por lo cual la frontera de producción del año 2007 retrocedió con respecto a la del 2006 y explica, en parte, el regreso tecnológico reportado. Otra parte puede ser expli- cada por el hecho de que la actividad agraria está fuertemente condicionada por variables genéticas, ambientales, administrativas y económicas, entre otras, algunas de los cuales han sido estudiadas en la ganadería cubana (Benítez et al., 2002). Los resultados promedios obtenidos pueden ser matizados por el hecho de que los valores del IPM y sus componentes del año 2007 determinaron los resultados promedios del periodo 2006-2008, a pesar de que en el último año estudiado hubo una ligera recuperación, lo que amerita extender la investigación a un periodo mayor, para minimizar los efectos de los factores coyunturales sobre la productividad. 


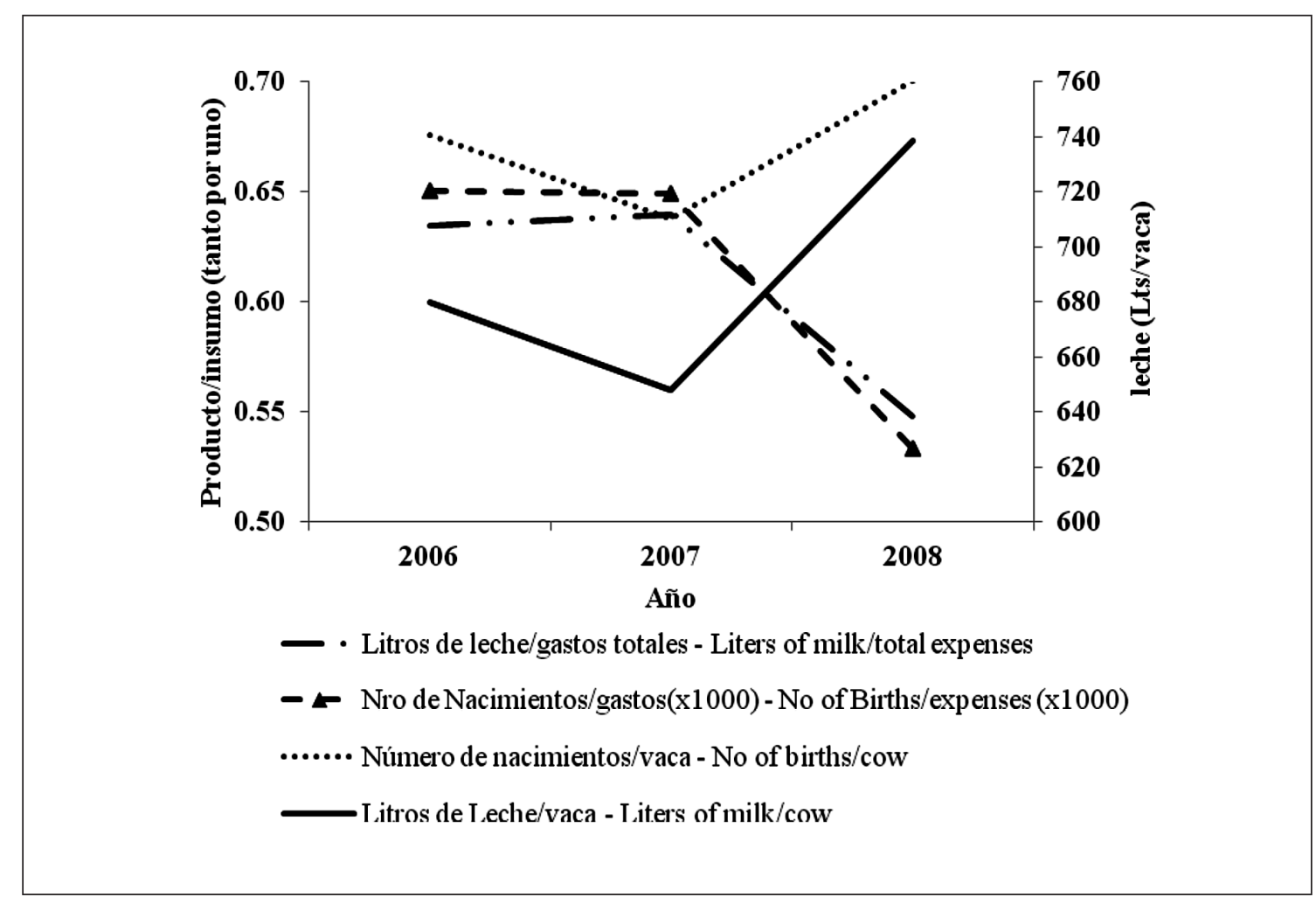

Figura 2. Comportamiento de la productividad de 24 explotaciones lecheras durante el periodo 2006-2008.

Figure 2. Productivity performance of 24 dairy farms in 2006-2008.

Asimismo, valores del IPM que reflejen caídas o incrementos promedios muy pequeños de la productividad son perfectamente viables en la ganadería cubana, ya que diversos obstáculos impiden el desarrollo eficiente de la producción de leche en el trópico (Nicholson, 1998; Osorio, 2001). Ello ha motivado el direccionamiento de un proceso de cambio dirigido a elevar los niveles actuales de producción y a reducir los costos, a partir de la utilización de sistemas basados en el uso de los recursos disponibles para las fincas lecheras en Cuba (Cino et al., 2004).

Debido a que los gastos totales de 2007 y 2008 fueron deflactados (expresados a precios corrientes del año 2006), se infiere que la uti- lización de los insumos que recoge esta cuenta se elevó durante el último periodo de estudio, pero fue apenas compensado por el crecimiento de la producción y, por ello, el IPM del último periodo fue positivo (aunque muy pequeño). También es posible que el deflactor no recoja todo el aumento de costos de los insumos (lo que es común en Latinoamérica) y entonces, la cuenta gastos totales deflactada también podría reflejar algún nivel de inflación en los precios de los insumos. El tipo de índice para deflactar es considerado un punto controvertido (Jan, 2011).

En virtud de los resultados antes mencionados, se puede concluir lo siguiente: 
1. Hubo una ligera caída de la productividad de las explotaciones lecheras durante el periodo 2006 al 2008. Al comparar los cambios en la productividad y sus componentes en 24 unidades lecheras ubicadas en el municipio San José de las Lajas (Cuba), durante los periodos 2006 al 2007 y 2007 al 2008 destaca que el descenso en la productividad total del primer periodo $(1,4 \%)$ no fue totalmente contrarrestado por un ligero aumento del $0,7 \%$ en el segundo periodo considerado, por lo cual, en promedio, hubo una pequeña caída de la productividad $(0,4 \%)$ entre el 2006 y el 2008.

2. La caída de la productividad de las explotaciones lecheras durante el periodo 2006 al 2008 fue ocasionada por un ligero retroceso tecnológico. Al indagar en las causas de este resultado mediante el examen de sus componentes, se evidencia que hubo mejora (aunque muy pequeña) en el segundo periodo con respecto al primero en el cambio de eficiencia técnica, que contribuyó a que el promedio fuese positivo de 1,2\%. El regreso tecnológico se acentúo ligeramente en el segundo periodo con respecto al primero, por lo cual, el promedio indica un ligero retroceso tecnológico de 1,6\%. El cambio favorable en la eficiencia se debió a una ligera mejora en cuanto a la escala de producción $(0,8 \%)$ y en el uso de la tecnología disponible $(0,5 \%)$. Estos valores promedios son los que caracterizan la productividad del sistema estudiado. Es importante destacar que el mencionado regreso tecnológico se debió, en parte, a un aumento en el número de vacas en el año 2007 que no se reflejó proporcionalmente en la producción de leche y en los nacimientos de becerros de ese año, lo cual abatió la relación de conversión producto-insumo, afectando a todas las fincas, incluyendo a las que estaban en la frontera de producción.
3. Se requieren prácticas gerenciales de alto impacto para mejorar la productividad del sistema ganadero estudiado. Para incrementar la productividad ganadera en las fincas evaluadas es necesario desarrollar y ejecutar estrategias para elevar la eficiencia de escala y la eficiencia técnica pura, así como inducir cambios tecnológicos. Para ello se deberían incrementar los niveles de productos (producción de leche por vaca y número de nacimiento de becerros por finca), para lo cual se pueden realizar entre otras, las actividades siguientes: 1) mejorar la tecnología adquiriendo, por ejemplo, nuevo equipamiento e insumos, implementar programas de mejoramiento genético, entre otras inversiones en activos, 2) Invertir en la capacitación del recurso humano a fin de optimizar el uso tanto de la nueva tecnología agraria como de la que ya está disponible, a fin de mejorar la eficiencia técnica, 3) Optimizar los costos de producción y demás insumos, 4) Promover la eficiencia de escala en aquellas fincas que operan a tamaños subóptimos y 5) formular planes de mejoramiento de la producción que incluyan metas. En general, para mejorar las explotaciones pecuarias se pueden seguir las orientaciones de González y Careaba (2004) y Senra et al. (2010), entre otros autores.

\section{Bibliografía}

Afriat S (1972). Efficiency Estimation of production Functions. International Economic Review 13: 568-598.

Arzubi A, Berbel J (2002). Determinación de índices de eficiencia mediante DEA en explotaciones lecheras de Buenos Aires. Investigación Agraria. Producción y Sanidad Animales 17(1-2): 104-123.

Banker R, Charnes A, Cooper W (1984). Some models for estimating technical and scale ineffi- 
ciencies in data envelopment analysis. Management Science 30(9): 1078-1092.

Benítez D, Ray J, Torres V, Ramírez A, Viamontes M, Tandrón I, Díaz M, Guerra J, Pérez D (2002). Factores determinantes en la eficiencia productiva de los rebaños de fincas ganaderas en sistemas de doble propósito en el Valle del Cauto (Granma), Cuba. Revista Cubana de Ciencia Agrícola 36(3): 217-222.

Brummer B, Glauben T, Thijssen G (2002). Decomposition of productivity growth using distance function: the case of dairy farms in three European countries. American Journal of Agricultural Economics 84:628-644.

Camp R (2001). Benchmarking, La búsqueda de las mejores prácticas de la industria que conducen a un desempeño excelente. Panorama Editorial S.A, México, $30 \mathrm{pp}$.

Canós L, Canós M, Sala R (2010). Productividad y eficiencia en la Liga Española de Fútbol (2002/032009/10), XVIII Jornadas ASEPUMA - VI Encuentro Internacional. Anales de ASEPUMA n ${ }^{\circ} 18$ : 509, Santiago de Compostela, España, 1-27 pp.

Caves D, Christensen L, Diewert E (1982). The Economic Theory of Index Numbers and the Measurement of Input, Output, and Productivity. Econométrica 50(6): 1393-1414.

Cino D, Martín P, Torres V (2004). Estudio económico preliminar de alternativas de producción de leche bovina. Revista Cubana de Ciencia Agrícola 38(1): 3-11.

Coelli T (1996). A Guide to DEAP Version 2.1: A Data Envelopment Analysis (Computer) Program. CEPA Working Paper No. 8/96. Universidad de Nueva Inglaterra, 50 pp.

Coelli T, Prasada-Rao D, Battesse G (1998). An Introduction to Efficiency and Productivity Analysis. Kluwer Academic Publishers, Boston.

Coelli T, Rao D, O'Donnell C, Battese G (2005). An introduction to efficiency and productivity analysis. 2da Ed. Springer, New York, USA.

Coll-Serrano V, Blasco-Blasco O, Cuñat-Giménez R (2009). Análisis de la eficiencia y evolución de la productividad de las cooperativas españolas en el periodo 1996-2004. Cayapa 9(18): 32-51.
Färe R, Grosskopf S, Lindgren B, Roos P (1989). Productivity developments in Swedish hospitals: A Malmquist output index approach. En Charnes A, Cooper WW, Lewin A, Seiford L (eds.) Data Envelopment Analysis: Theory, methodology and applications, Kluwer Academic Publishers, Boston.

Färe R, Grosskopf S, Norris M, Zhang Z (1994). Productivity Growth, Technical Progress, and Efficiency Change in Industrialized Countries. American Economic Review 84(1): 66-83.

Färe R, Grosskopf S, Roos P (1998). Malmquist productivity indexes: a survey of theory and practice. En Färe R, Grosskopf S, Russell R (Eds.). Index Numbers: Essays in Honour of Sten Malmquist. Kluwer Academic Publishers, Norwell, MA.

Färe R, Grosskopf S, Lindgren B, Roos P (1992). Productivity Change in Swedish Pharmecies 19801989: A Nonparametric Malmquist Approach. Journal of Productivity Analysis 3: 85-102.

Färe R, Grosskopf S, Norris M (1994). Productivity growth, technical progress and efficiency change in industrialized countries. American Economic Review 84: 66-83.

Färe R, Grosskopf S, Norris M (1997). Productivity growth, technical progress, and efficiency change in industrialized countries: reply, American Economic Review 87 (5): 1040-1043.

Ferro G, Romero C (2011). Comparación de medidas de cambio de productividad. Las aproximaciones de Malmquist y Luenberger en una aplicación al mercado de seguros. Disponible En: http://hal.archives-ouvertes.fr/docs/00/59/79/ 46/PDF/Ferro_Romero_-_AAEP_180511_documento_de_trabajo.pdf (22 de Marzo 2012)

González A (2009). Tenemos que dar un salto cualitativo. Entrevista a Orlando Lugo Fonte. Trabajadores, $6 \mathrm{pp}$.

González E, Careaba A (2004). Efficiency improvement through learning. International Journal of Technology Management 27(6-7): 628-638.

Graham M (2009). Developing a Social Perspective to Farm Performance Analysis. Ecological Economics 68: 2390-2398. 
Gray E, Sheng Y, Nossal K, Oss-Emer M, Davidson A (2011). Improving productivity - the incentives for change. Australian Commodities 18: 218-234.

Gregg D, Rolfe J (2011). Identifying sources and trends for productivity growth in a sample of Queensland broad-acre beef enterprises. Animal Production Science 51: 443-453.

Grosskopf S (1993). Efficiency and productivity. En: Fried H., Lovell K. y Schmidt S. (Eds.). The measurement of productive efficiency. Techniques and applications. Oxford University Press, 175, 428 pp.

Jan P (2011). Total Factor Productivity Change of the Swiss Dairy Sector for the Mountain Region in the Period 1999 to 2008, 2011 International Congress, Zurich, Suiza, pp. 1-16.

Karanja F, Gilmour D, Fraser I (2012). Dairy productivity growth, efficiency change and technological progress in Victoria. En: 2012 annual conference of the Australian Agricultural and Resource Economics Society. Fremantle, Australia Occidental, pp. 1-24.

Kompas T, Che T. 2004. Productivity in the Australian Dairy Industry. Australasian Agribusiness Review, vol. 12, no. 1-30, pp. 1-28.

Latruffe L, Fogarasi J (2009). Farm performance and support in Central and Western Europe: A comparison of Hungary and France. Working Paper SMART-LERECO Nº 09-07, 1-16 pp.

Lerdon J, Muñoz C, Moreira V (2010). Caracterización económico-productiva de medianos y grandes productores de leche del sur de Chile. IDESIA 28(3): 41-49.

Li L, Tsunekawa A, Tsubo M, Koike A, Wang J (2010). Assessing total factor productivity and efficiency change for farms participating in Grain for Green program in China: A case study from Ansai, Loess Plateau. Journal of Food, Agriculture \& Environment 8(2): 1185-1192.

Luik H, Omel R, Viira A (2011). Efficiency and productivity change of Estonian dairy farms from 2001-2009. Estonian University of Life Sciences. República de Estonia.
Malmquist S (1953). Index Numbers and Indifference Surfaces. Trabajos de Estadística 4: 209-242.

Maroto A (2007). La productividad en el sector servicios. Un análisis económico aplicado. Tesis Doctoral. Facultad de Ciencias Económicas y Empresariales. Departamento de Economía Aplicada. Universidad de Alcalá, España, 110 pp.

Martín M (2000). Eficiencia y progreso técnico en el sistema portuario español, III Encuentro de economía aplicada, Valencia, España.

Mirabal M (2010). Fomento de la base nacional forrajera: premisa fundamental para la recuperación de la ganadería vacuna. Tesis en opción al título de Licenciada en Economía. Facultad de Economía, Universidad de La Habana, Cuba, 78 pp.

Miranda T, Machado H, Suárez J, Sánchez T, Lamela L, Iglesia J, Suset A, Pérez A, Milera M, Martín G, Campo M, López O, Sánchez S, Simón L (2012). La innovación y la transferencia de tecnologías en la Estación Experimental "Indio Hatuey": 50 años propiciando el desarrollo del sector rural cubano (Parte II). Pastos y Forrajes 35(1): 3-16.

Mkhabela T (2011). An Econometric Analysis of the Economic and Environmental Efficiency of Dairy Farms in the KwaZulu-Natal Midlands. Tesis doctoral. Stellenbosch University, Sudáfrica, $11 \mathrm{pp}$.

Moreira-López V, Bravo-Ureta B, Arzubi A, Schilder E (2006). Multi-output technical efficiency for Argentinean dairy farms using stochastic production and stochastic distance frontiers with unbalanced data. Economía Agraria 10: 97-106.

Nicholson C (1998). Intensificación de sistemas de producción bovina en los trópicos americanos: su impacto social y ambiental. Archivos Latinoamericanos de Producción Animal 6: 1-18.

Nova A (2008). El sector agropecuario en Cuba. Nueva Sociedad 216: 77-89.

O’Donnell C (2009). An aggregate quantity-price framework for measuring and decomposing productivity and profitability change. Centre for Efficiency and Productivity Analysis, Brisbane.

O'Donnell C (2010). Measuring and decomposing agricultural productivity and profitability change. Australian Journal of Agricultural and Resource Economics 54: 527-560. 
Oficina Nacional de Estadísticas de Cuba (2012). Índices de precios. Cuentas nacionales. Anuario estadístico de Cuba 2010. Disponible en: http:// www.one.cu/aec2010/esp/05_tabla_cuadro.htm (12 agosto 2012).

Osorio J (2001). Costos de producción y productividad de lecherías especializadas del trópico alto en Colombia. En: Costos e Insumos. Documentos de Trabajo, Corpoica, 28 pp.

Pastor J (1994). Determinantes de la dinámica de la productividad de los bancos y cajas de ahorro españolas. WP-EC 94-07. Instituto Valenciano de Investigaciones Económicas, Universidad de Valencia, España.

Pastor J (1995). Eficiencia, cambio productivo y cambio técnico en los Bancos y Cajas de Ahorro españolas: Un análisis de la frontera no paramétrico. Universitat de València. WP-EC 95-09. 12 pp.

Ponce P (2009). Un enfoque crítico de la lechería internacional y cubana. Rev Salud Anim 31(2): 77-85.

Quirós C, Picazo A (2001). Liberalización, eficiencia y cambio tecnológico en comunicaciones, Revista de Economía Aplicada 9(25): 77-113.

Ray S, Desli E (1997). Productivity Growth, Technical Progress, and Efficiency Change in Industrialized Countries: Comment. American Economic Review 87(5):1033-1039.

Schuschny A (2007). El método DEA y su aplicación al estudio del sector energético y las emisiones de CO2 en América Latina y el Caribe. CEPAL, Santiago de Chile, 22 pp.

Senra A, Soto S, Guevara R (2010). Guía estratégica sobre la base de reservas en alternativas de la ganadería cubana, para enfrentar la crisis económica global y el cambio climático. Avances en Investigación Agropecuaria 14(3): 3-18.

Simar L, Wilson P (1999). Estimating and bootstrapping Malmquist indices. European Journal of Operations Research 115: 459-471.

Singh P, Singh A (2012). Decomposition of Technical Change and Productivity Growth in Indian Agriculture Using Non-Parametric Malmquist Index. Eurasian Journal of Business and Economics 5(9): 187-202.

Tauer L (1998). Productivity of New York dairy farms measured by non parametric Malmquist indices. Journal of Agricultural Economics 49(2): 234-249.

Tone K (2004). Malmquist Productivity Index: Efficiency Change Over Time. En: Cooper W, Seiford L, Zhu J (Eds.): Handbook on Data Envelopment Analysis. Kluwer Academic Publishers, Norwell, MA.

Toro P, García A, Aguilar C, Acero R, Perea J, Vera $R$ (2010). Determinación de la eficiencia técnica en agroecosistemas. Documentos de trabajo. Dpto de Producción Animal y Gestión, Universidad de Córdoba 2: 1-17.

Zuniga C (2010). Impacto de los Sistemas de Producción Agropecuarios en el Desarrollo Local Sostenible de Nicaragua (1998-2005: Índice de Malmquist DEA con un Output Orientado). Tesis de Maestría. Universidad Nacional Autónoma de Nicaragua, 40 pp.

(Aceptado para publicación el 28 de mayo de 2013) 